\title{
Sinnfindung, Krankheitsverarbeitung und Lebensqualität von Tumorpatienten im perioperativen Verlauf
}

\author{
Inauguraldissertation \\ zur Erlangung des Doktorgrades der Philosophie \\ am Fachbereich Erziehungswissenschaft und Psychologie \\ der Freien Universität Berlin
}

eingereicht von

Steffen Taubert

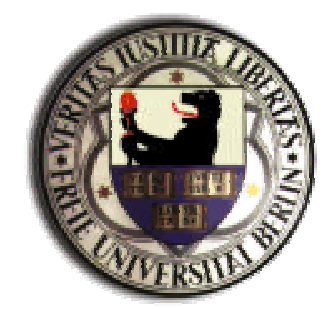

Disputation: 23.04.2003

\section{Gutachter}

Prof. Dr. Ralf Schwarzer, Freie Universität Berlin

Prof. Dr. Dieter Kleiber, Freie Universität Berlin 

gewidmet Charis, Anthony \& Alischa

Bedanken möchte ich mich bei Herrn Prof. Dr. Ralf Schwarzer für die Betreuung und bei Prof. Dr. Dieter Kleiber für die Begutachtung der Arbeit. Besonderen Dank gilt den Studienteilnehmern und Projektmitarbeitern, ohne die diese Arbeit nicht möglich gewesen wäre. Für wertvolle Hinweise bei der Erstellung der Arbeit möchte ich mich bei Dr. Judith Bäßler, Dr. Nina Knoll, Dr. Britta Renner, Friederike Kendel und Charis Förster bedanken. 



\section{Inhalt Kapitel}

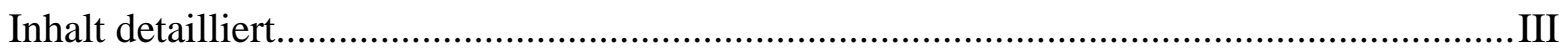

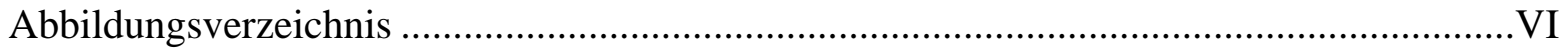

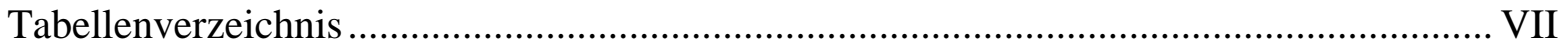

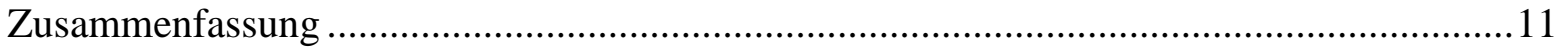

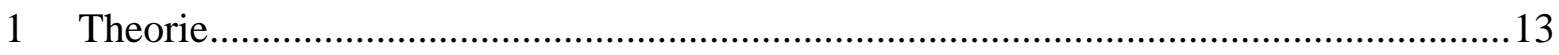

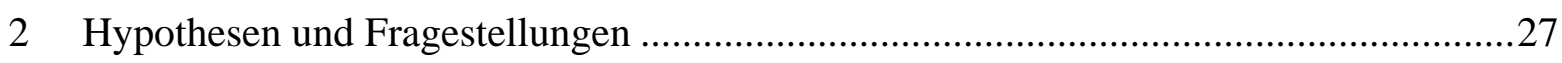

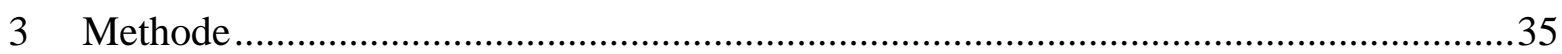

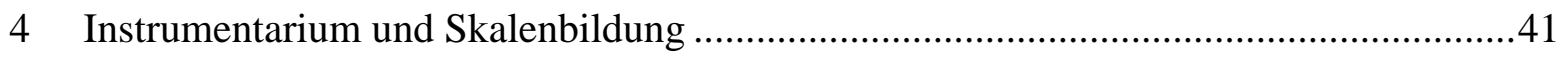

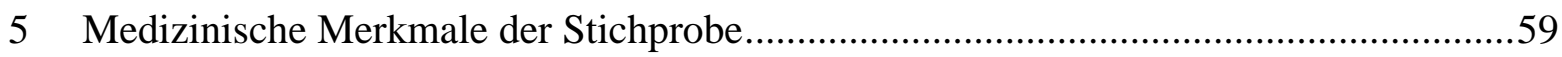

6 Veränderungsverläufe und Korrelationen von Krankheitsverarbeitung, emotionalem Wohlbefinden und gesundheitsbezogener Lebensqualität ........................................ 77

7 Veränderung von Sinnfindung im Krankheitsverlauf .............................................93

8 Vorhersage von Wohlbefinden und Lebensqualität durch die Veränderung von

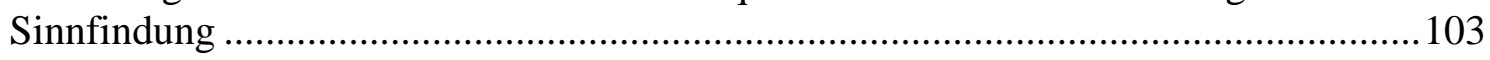

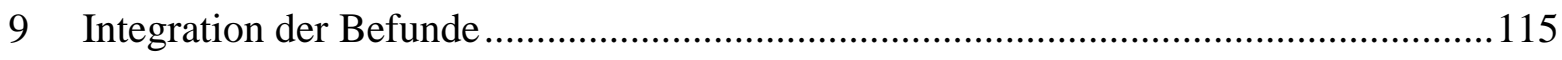

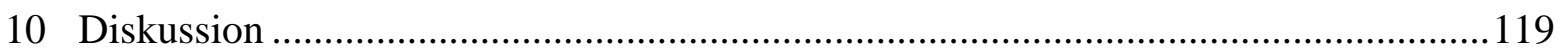

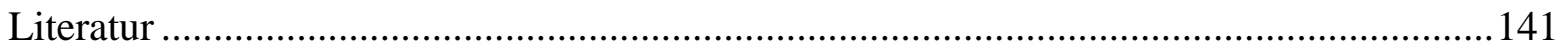

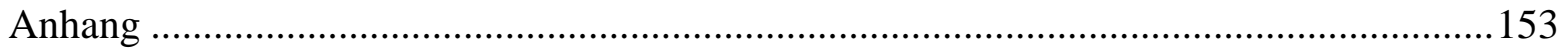

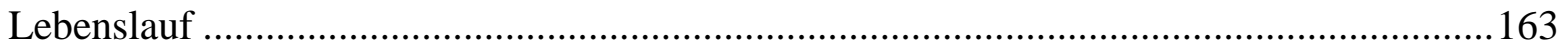

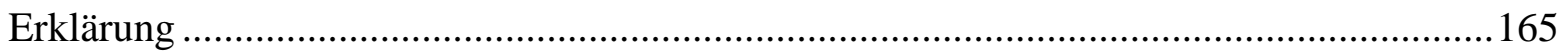





\section{Inhalt detailliert}

Inhalt detailliert

Tabellenverzeichnis

Zusammenfassung

1 Theorie

$1.1 \quad$ Einleitung: Sinnfindung und die kognitiv-transaktionale Theorie der Stressbewältigung .......................13

1.1.1 Der Stressor: Die Belastung durch die Tumorerkrankung ................................................... 14

1.1.2 Individuelle Bewertung der Krebserkrankung ............................................................. 17

1.1.3 Problembezogene und emotionsregulierende Krankheitsverarbeitung ........................................18

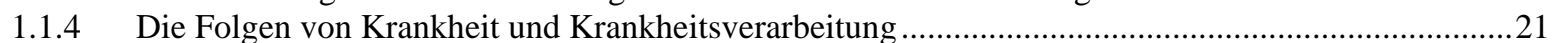

1.1.5 Sinnfindung als Krankheitsverarbeitung im kognitiv-transaktionalen Stressbewältigungsmodell ......22

1.1.6 Wohlbefinden und gesundheitsbezogene Lebensqualität bei Tumorpatienten ...............................24

1.1.7 Prozesscharakter von Krankheit und Krankheitsverarbeitung ..................................................26

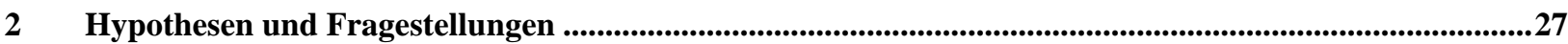

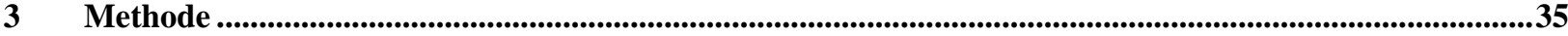

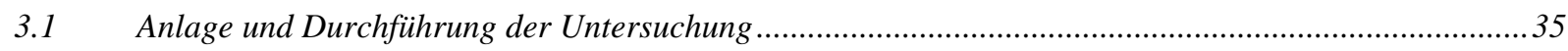

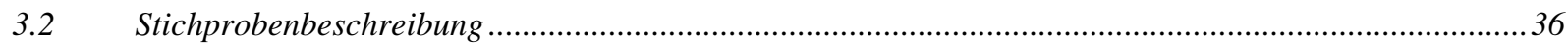

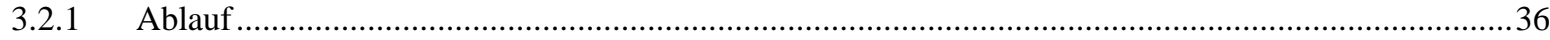

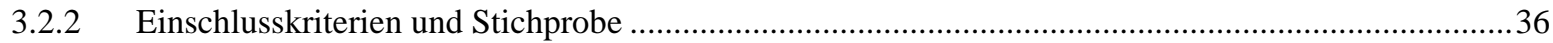

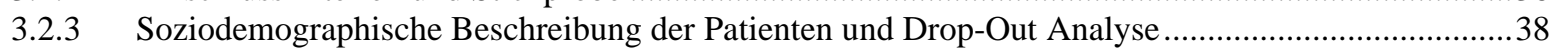

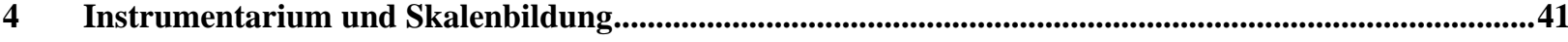

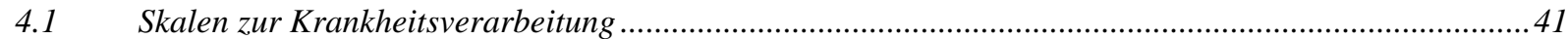

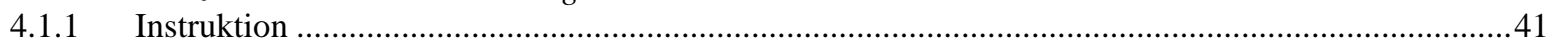

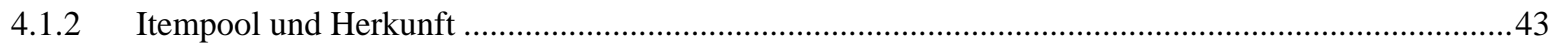

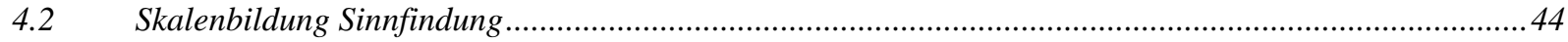

4.3 Skalenbildung Problembezogene und Emotionsregulierende Krankheitsverarbeitung ...........................47

4.4 Skalen und Indikatoren für emotionales Wohlbefinden und gesundheitsbezogene Lebensqualität...........52

4.4.1 Emotionales Wohlbefinden: Depressivität .............................................................................52

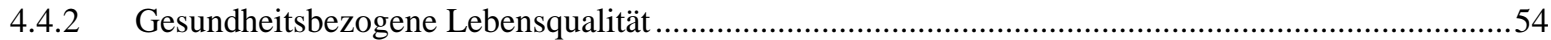

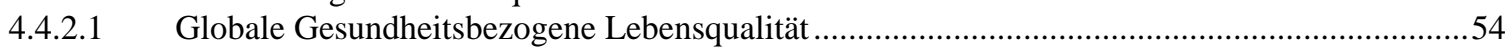

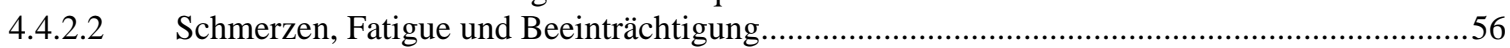

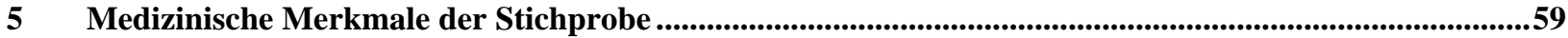

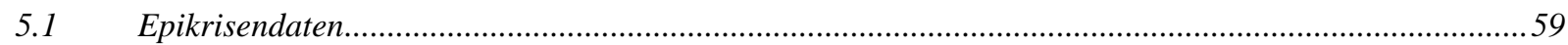

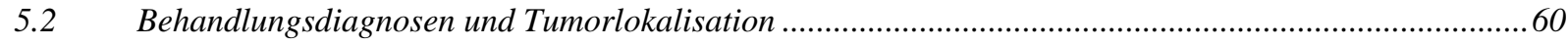

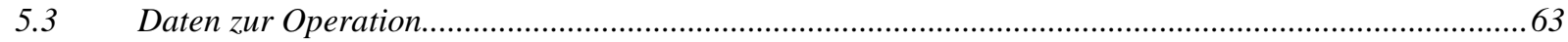

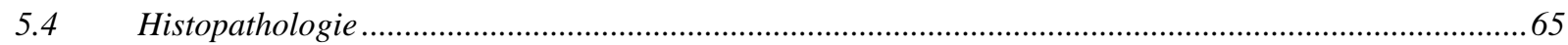

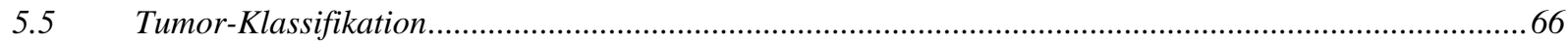

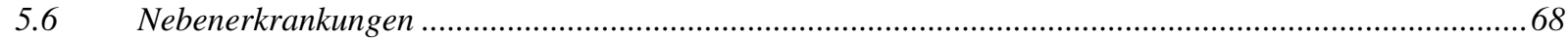

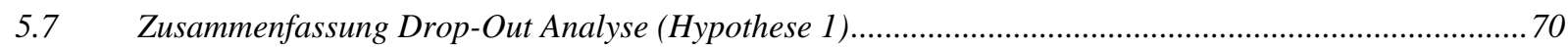

5.8 Zusammenhang Medizinische Parameter, Krankheits-verarbeitung und Wohlbefinden .......................71 
6 Veränderungsverläufe und Korrelationen von Krankheitsverarbeitung, emotionalem Wohlbefinden und gesundheitsbezogener Lebensqualität ..................................................................................................77

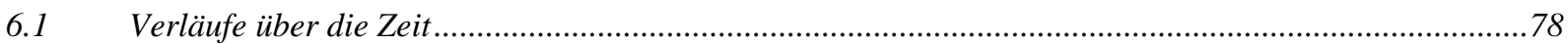

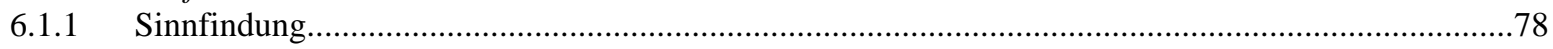

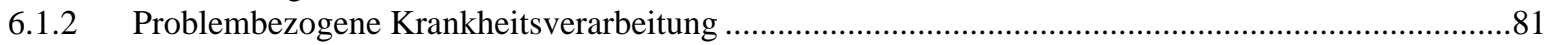

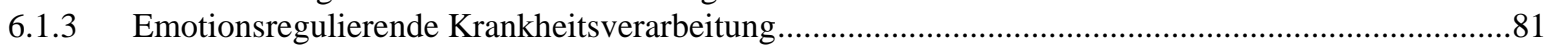

6.1.4 Verläufe Sinnfindung, problembezogene und emotionsregulierende Krankheitsverarbeitung im

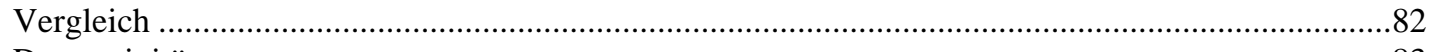

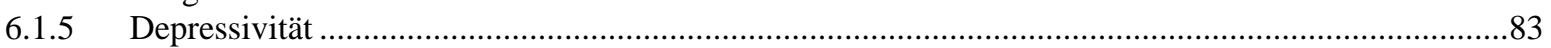

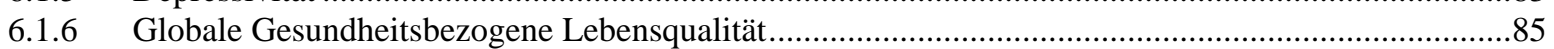

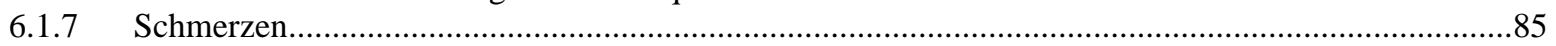

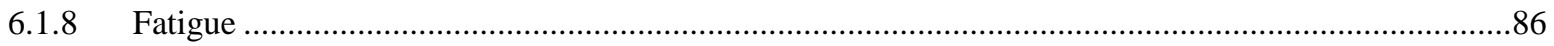

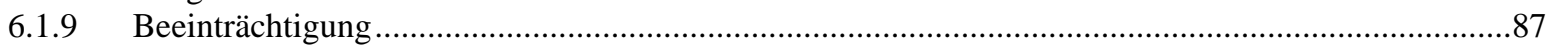

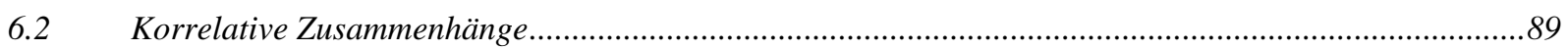

6.2.1 Zusammenhang Sinnfindung, problembezogene und emotionsregulierende Krankheitsverarbeitung 89

6.2.2 Zusammenhang Krankheitsverarbeitung, emotionales Wohlbefinden und gesundheitsbezogene

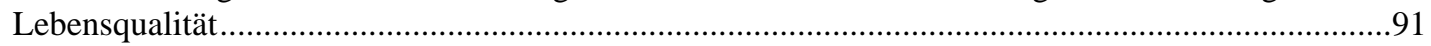

7 Veränderung von Sinnfindung im Krankheitsverlauf ............................................................................93

7.1 Differenzmaß oder residualisiertes Veränderungsmaß? .....................................................................93

7.2 Bildung von Differenzmaßen und deren Verteilungseigenschaften ......................................................95

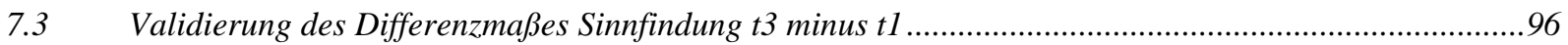

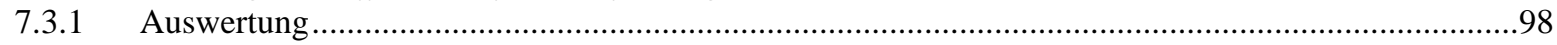

7.3.2 Altersgruppen- und Geschlechtszugehörigkeit …………...........................................................99

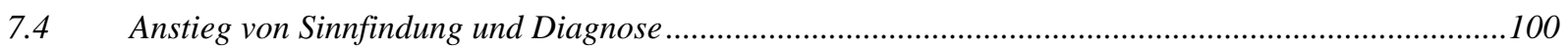

8 Vorhersage von Wohlbefinden und Lebensqualität durch die Veränderung von Sinnfindung...............103

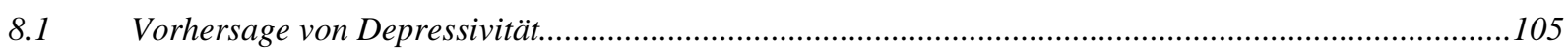

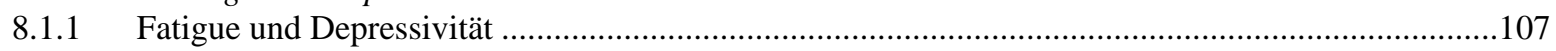

8.2 Vorhersage von Globaler Gesundheitsbezogener Lebensqualität......................................................110

8.3 Vorhersage von Schmerzen, Fatigue und Beeinträchtigung............................................................112

$9 \quad$ Integration der Befunde ...............................................................................................................................115

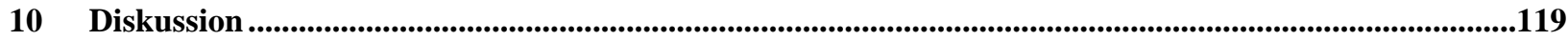

$10.1 \quad$ Krankheitsbezogener Ereignishintergrund …….....................................................................119

$10.2 \quad$ Schweregrad, Wohlbefinden und Krankheitsverarbeitung ..............................................................121

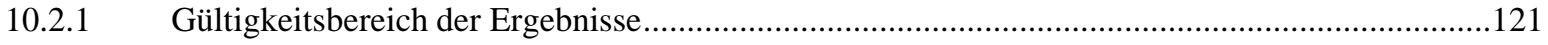

10.2.2 Schweregrad der Tumorerkrankung und Wohlbefinden ..........................................................122

10.2.3 Schweregrad der Tumorerkrankung und Krankheitsverarbeitung ...............................................122

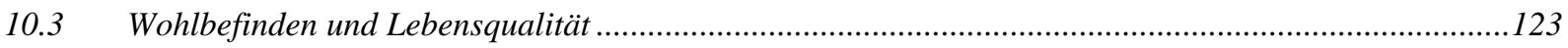

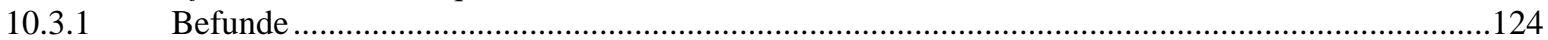

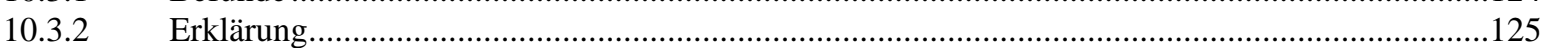

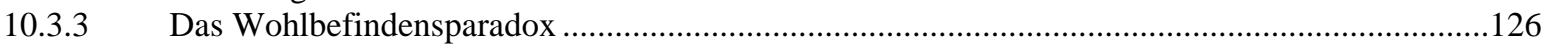

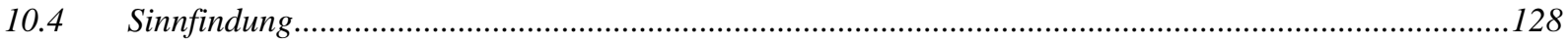

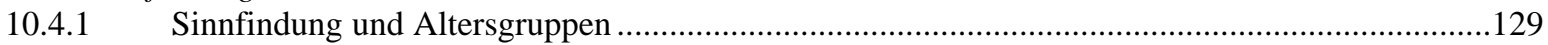

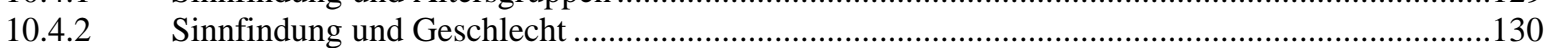

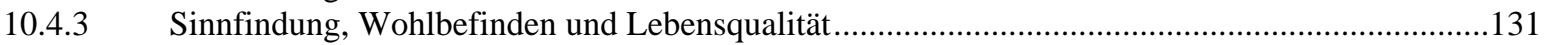

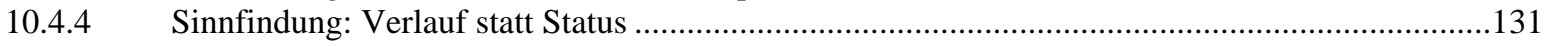

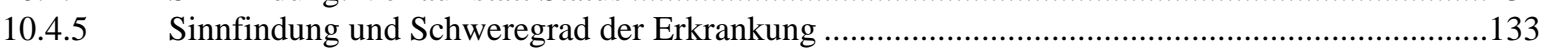

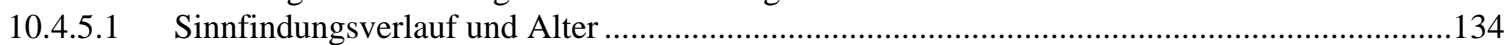




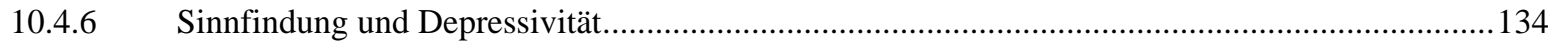

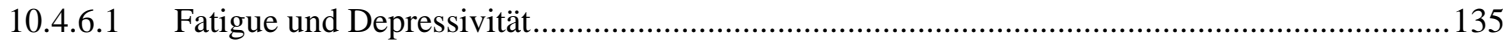

10.4.7 Sinnfindung, problembezogene und emotionsregulierende Krankheitsverarbeitung...................136

10.4.7.1 Emotionsregulierende Krankheitsverarbeitung ..............................................................136

10.4.7.2 Problembezogene Krankheitsverarbeitung .................................................................. 137

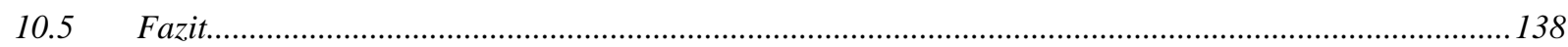

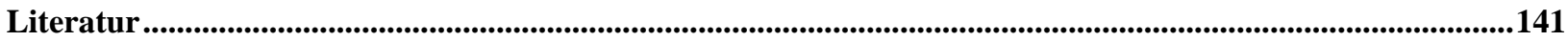

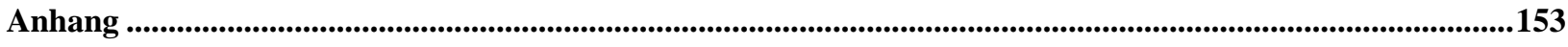

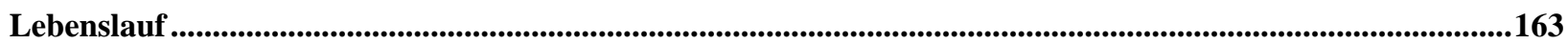

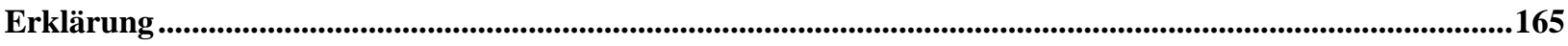




\section{Abbildungsverzeichnis}

Abbildung 1. Das adaptierte kognitiv-transaktionale Stressbewältigungsmodell. ...................... 14

Abbildungen 2a bis d. Verteilung der Variable Sinnfindung zu vier Messzeitpunkten. ............... 47

Abbildungen 3a bis d. Verteilung Depressivität zu vier Messzeitpunkten.................................. 54

Abbildungen 4a bis d. Verteilung Globale Gesundheitsbezogene Lebensqualität zu vier

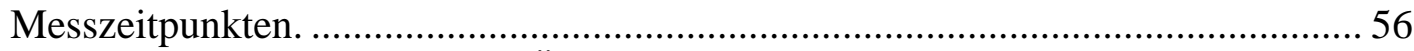

Abbildung 5. Deutschlandweite Fünf-Jahres-Überlebensrate in Prozent nach

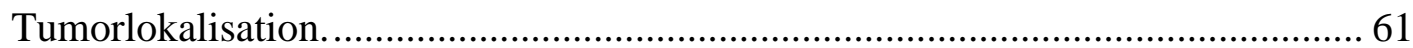

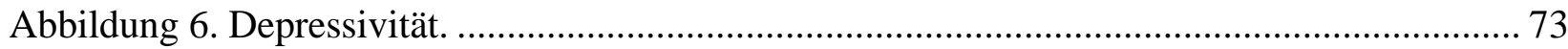

Abbildung 7. Globale Gesundheitsbezogene Lebensqualität. .................................................. 73

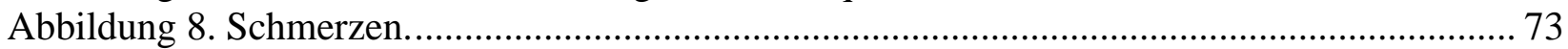

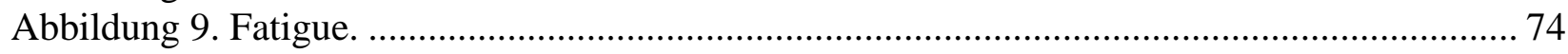

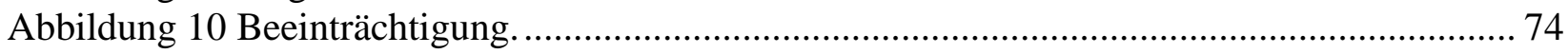

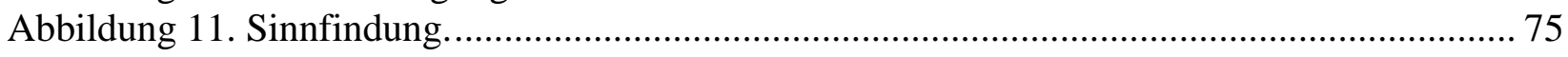

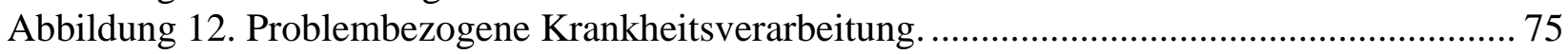

Abbildung 13. Emotionsregulierende Krankheitsverarbeitung. …........................................... 75

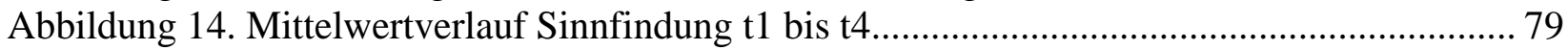

Abbildung 15. Mittelwertverlauf Sinnfindung t1 bis t4 nach drei Altersgruppen....................... 80

Abbildung 16. Mittelwertverlauf Sinnfindung t1 bis t4 nach Geschlecht. ................................. 80

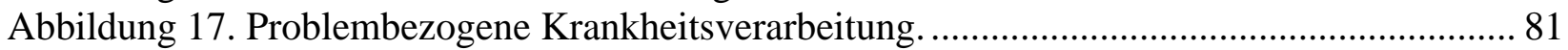

Abbildung 18. Emotionsregulierende Krankheitsverarbeitung. …............................................ 82

Abbildung 19. Sinnfindung, Problembezogene und Emotionsregulierende

Krankheitsverarbeitung im Vergleich. ........................................................ 83

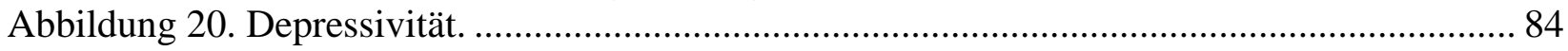

Abbildung 21. Depressivität nach Geschlecht. ...................................................................... 84

Abbildung 22. Mittelwertverlauf Depressivität nach drei Altersgruppen. ................................ 84

Abbildung 23. Globale Gesundheitsbezogene Lebensqualität. ............................................. 85

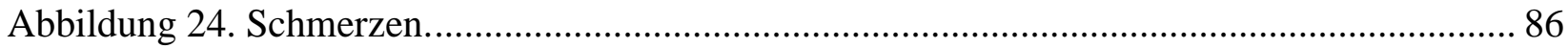

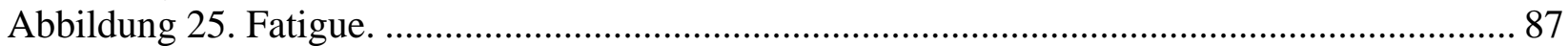

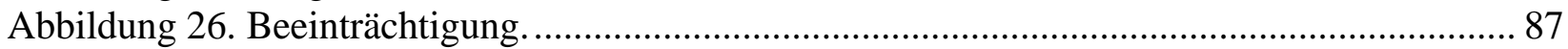

Abbildungen 27 a bis c. Fatigue, Alterskohorte und Geschlecht............................................... 88

Abbildung 28. Korrelationen Differenzmaß Sinnfindung t 3 minus $t 1$ mit Wohlbefinden und Lebensqualität zu t4 nach Altersgruppen.

Abbildung 29. Mittelwerte des Differenzmaßes von Sinnfindung t3 minus t 1 und t 4 minus t3

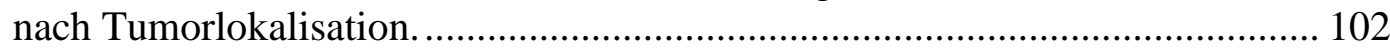

Abbildung 30. Multiples Regressionsmodell zur Vorhersage von Depressivität. ...................... 104

Abbildung 31. Multiple Regression zur Vorhersage von Depressivität. ................................... 106

Abbildung 32. Differenzmaß t3 minus t1 von Fatigue nach medizinischer Folgebehandlung zu

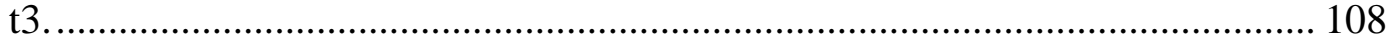

Abbildung 33. Multiple Regression zur Vorhersage von globaler gesundheitsbezogener

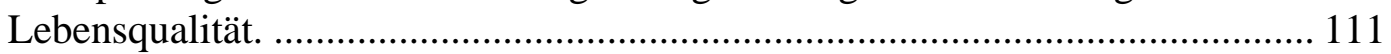

Abbildung 34. Multiple Regression zur Vorhersage von Beeinträchtigung.............................. 113

Abbildung 35. Ausgangsmodell: Vorhersage von Depressivität und Globaler Gesundheitlicher

Lebensqualität durch Krankheitsverarbeitung.

Abbildung 36. Endgültiges Pfadmodell zur Vorhersage von Depressivität und Globaler

Gesundheitlicher Lebensqualität durch Krankheitsverarbeitung (standardisierte

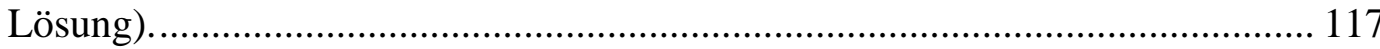

Abbildung 37a bis d. Screeplotts Hauptkomponentenanalyse Sinnfindung.............................. 153 


\section{Tabellenverzeichnis}

Tabelle 1 Erlebte oder befürchtete Belastungen durch eine Krebserkrankung und

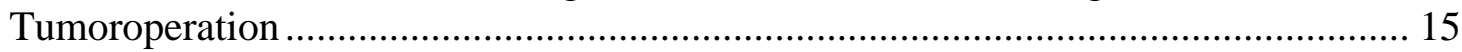

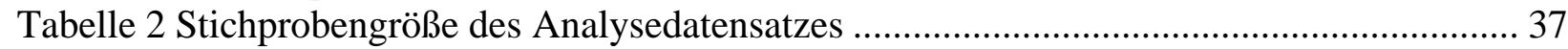

Tabelle 3 Soziodemographische Beschreibung der Stichprobe .................................................. 39

Tabelle 4 Überschriften und Instruktionen zum Fragebogenteil Krankheitsverarbeitung............ 42

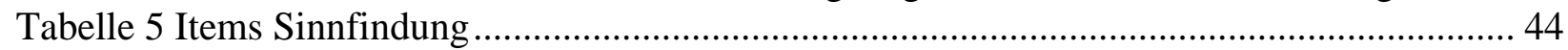

Tabelle 6 Hauptkomponentenanalyse Sinnfindung: Ladungen und Kommunalitäten ................ 46

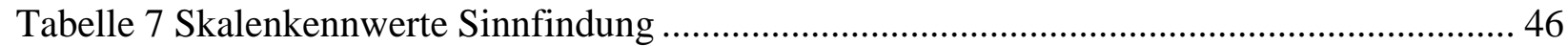

Tabelle 8 Faktorladungen des finalen Modells: Sinnfindung, emotionsregulierende und problembezogene Krankheitsverarbeitung............................................................. 50

Tabelle 9 Items problembezogene Krankheitsverarbeitung .................................................. 51

Tabelle 10 Items emotionsregulierende Krankheitsverarbeitung ............................................ 51

Tabelle 11 Skalenkennwerte problembezogene und emotionsregulierende

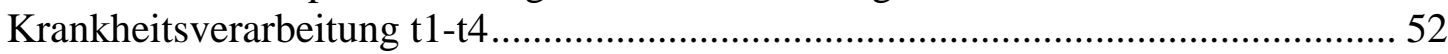

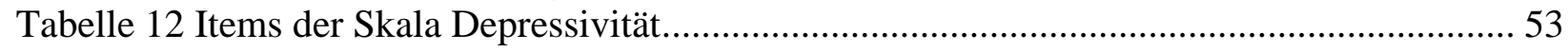

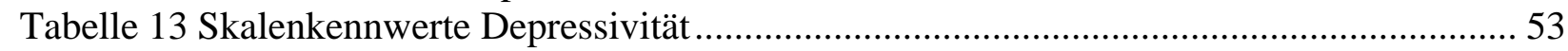

Tabelle 14 Skalenkennwerte Globale Gesundheitsbezogene Lebensqualität............................. 55

Tabelle 15 Items und Antwortformat spezifische gesundheitsbezogene Lebensqualität ............. 57

Tabelle 16 Itemkennwerte Schmerzen, Fatigue und Beeinträchtigung ...................................... 57

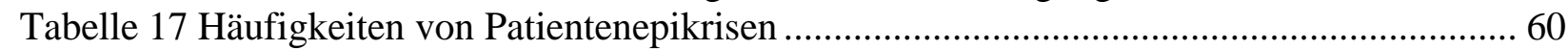

Tabelle 18 Häufigkeiten von Behandlungsdiagnosen im Gruppenvergleich ............................ 62

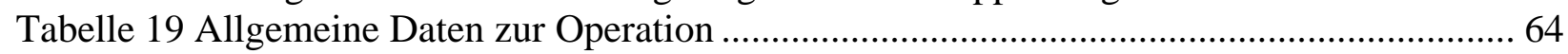

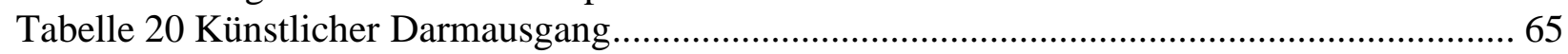

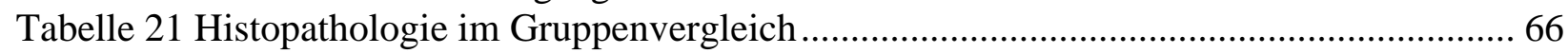

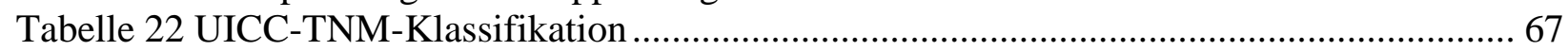

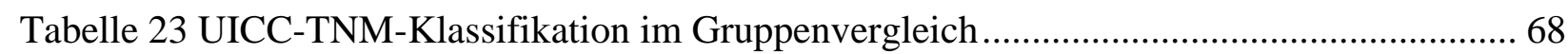

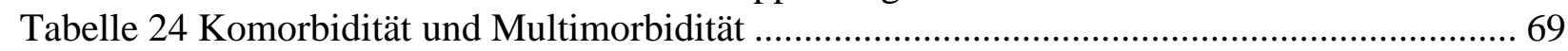

Tabelle 25 Korrelationen: Krankheitsverarbeitung und gesundheitsbezogene Lebensqualität im Quer- und Längsschnitt .................................................................................... 90

Tabelle 26 Kennwerte und Verteilung der Differenzmaße von Krankheitsverarbeitung und

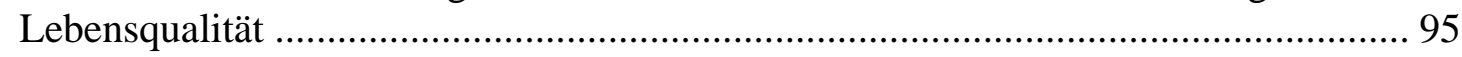

Tabelle 27 Korrelation des Differenzmaßes von Sinnfindung t 3 minus $t 1$ im Vergleich ............ 98

Tabelle 28 Korrelationen Differenzmaß Sinnfindung t3 minus t1 mit Wohlbefinden und Lebensqualität zu t4 nach Altersgruppen

Tabelle 29 Vorhersage von Depressivität t4 unter Kontrolle vom t1 Ausgangswert durch die

Differenzmaße von Krankheitsverarbeitung und Lebensqualität.............................. 106

Tabelle 30 Korrelation des Differenzmaßes t3 minus t1 von Fatigue mit Depression zu t4 nach

Altersgruppen und Geschlecht

Tabelle 31 Vorhersage von Globaler Gesundheitsbezogener Lebensqualität t 4 durch

Differenzwerte Krankheitsverarbeitung und Lebensqualität unter Kontrolle von t1 111

Tabelle 32 Vorhersage Beeinträchtigung unter Kontrolle der Ausgangswerte zu t1 durch

Krankheitsverarbeitung und Lebensqualität........................................................ 113

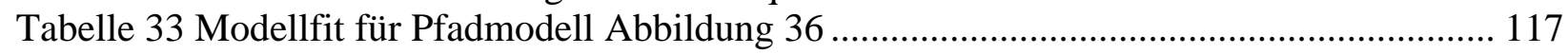

Tabelle 34 Faktorladungen des Ausgangsmodells Krankheitsverarbeitung............................. 154

Tabelle 35 Von der Skalenbildung ausgeschlossene Items ................................................... 155

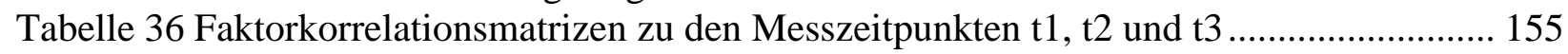


Tabelle 37 ANOVA mit Messwiederholung: Krankheitsverarbeitung und Lebensqualität im Verlauf .

Tabelle 38 ANOVA mit Messwiederholung: Polynomiale Kontraste für den Messwiederholungsfaktor. 157

Tabelle 39 ANOVA mit Messwiederholung: Paarweiser Vergleich von Differenzwerten nach Messzeitpunkten 158

Tabelle 40 ANOVA mit Messwiederholung: Abweichung (Kontraste) des Mittelwerts einzelner Messzeitpunkte vom Mittelwert aller Messzeitpunkte 160

Tabelle 41 t-Tests: Geschlechtsdifferenzen nach Messzeitpunkten $(n=84)$ 161

Tabelle 42 Vorhersage Schmerzen unter Kontrolle der Ausgangswerte zu t1 durch Krankheitsverarbeitung und Lebensqualität 162

Tabelle 43 Vorhersage Fatigue unter Kontrolle der Ausgangswerte zu t1 durch Krankheitsverarbeitung und Lebensqualität. 162 

